\title{
Research on Modeling for Administrative Law Enforcement Based on
}

\section{Workflow-net}

\author{
Zhenxin $Q u^{1, a}$, Zhanghui ${ }^{1, ~ b}$, Liping $\operatorname{Liu}^{2, c}$ \\ ${ }^{1}$ Information and Safety Engineering School, Zhongnan University of Economics and Law, Wuhan, \\ 430073, China \\ ${ }^{2}$ Propaganda department, Zhongnan University of Economics and Law, Wuhan, 430073, China \\ aquzx72@gmail.com, 'bhezi904@yahoo.com.cn, 'Ipliu@znufe.edu.cn
}

Keywords: model, enforcement, workflow-net

\begin{abstract}
To create simulation experimental software correctly, how to model for process of administrative law enforcement was discussed. Taking current regulations in China as example, two laws was modeled using workflow-net. Net for administrative reconsideration law enforcement was simplified, and then proved to be soundless. Improving method was proposed for program realization. The research shows that workflow-net model is necessary and effective for analysis of enforcement, which helps to find problem and improve it.
\end{abstract}

\section{Introduction}

Practice is necessary in teaching of administrative law enforcement, which helps students to learn and understand process of administrative law enforcement. Simulation experimental software is a good choice to reach it. Flow of administrative law enforcement will be expressed in the software, which illustrates how to enforce laws. To program, process of administrative law enforcement should be expressed in flowing way firstly. In fact, the work is to model for process of administrative law enforcement. Model described with standardized and formal method is easy to be verified and realized. Workflow-net technology is suit for modeling for process of administrative law enforcement.

Workflow-net is based on Petri net, supported by powerful mathematical theory, having intuitive graph, with which business process can be summarized and abstracted. Flow of business can be described accurately without ambiguity. There are also abundant technologies for analyzing and verifying workflow-net. Simulation tools for Petri net can also be used to analyze and optimize workflow model. In these ways, model can be adapted to best status.

In our project, we model for process of administrative law enforcement using workflow-net firstly. Models have accurate semantics and intuitive graphs. Later, we create programs realizing it using jBPM, popular work flow software.

\section{Workflow-net}

Workflow-net was proposed by Van der Aalst worked in Eindhoven University, which is on the basis of Petri net. The technology is suit for modeling for work flow.

Petri net[1] was proposed by Cal Adam Petri in 1962. It is a powerful tool for modeling and analyzing process, and describes process with graphical style. The technology is supported by powerful mathematical theory and used as formal description tool. 
Petri net model has four basic elements: place, transition, arc and token. Place is to describe possible local status of system. Transition is to describe event which can modify status of system. Arc links place and transition, which looks like a directed arrow. Token is to describe available data or resource, which is living in place. The Petri net is a directed bipartite graph.

Definition 1 (Petri net) A Petri net is a triple $\mathrm{PN}=(\mathrm{P}, \mathrm{T}, \mathrm{F})$ :

(1) $\mathrm{P} \cap \mathrm{T}=\varnothing$

(2) $\mathrm{P} \cup \mathrm{T} \neq \varnothing$

(3) $\mathrm{F} \subseteq(\mathrm{P} \times \mathrm{T}) \cup(\mathrm{T} \times \mathrm{P})$

(4) $\operatorname{dom}(\mathrm{F}) \cup \operatorname{cod}(\mathrm{F})=\mathrm{P} \cup \mathrm{T}$

$\operatorname{dom}(\mathrm{F})=\{\mathrm{x} \mid \exists \mathrm{y}:(\mathrm{x}, \mathrm{y}) \in \mathrm{F}\} \quad($ domain of $\mathrm{F})$

$\operatorname{cod}(F)=\{y \mid \exists x:(x, y) \in F\} \quad($ range of $F)$

$\mathrm{P}$ is set of places, $\mathrm{T}$ is set of transitions, and $\mathrm{F}$ is set of arcs that connect places and transitions.

Workflow-net[2] is Petri net that describes work flow. Model described with Petri net is intuitive. Task in work flow is denoted by transition of Petri net, condition in work flow is denoted by place, work flow instance is denoted by token, logical relation between tasks and circulation of work is denoted by arc, execution of workflow is denoted by flowing of token.

Definition 2 (Workflow-net) A Petri net $\mathrm{PN}=(\mathrm{P}, \mathrm{T}, \mathrm{F})$ is a workflow-net if and only if:

(1) PN has two special places: $\mathrm{i}$ and $\mathrm{o}$. Place $\mathrm{i}$ is a source place: $\cdot \mathrm{i}=\varnothing$. Place $\mathrm{o}$ is a sink place: $\cdot \mathrm{o}=\varnothing$.

(2) If we add a transition $t^{*}$ to $P N$ which connects place o with i (i.e. $\cdot t^{*}=\{0\}$ and $t^{*} \cdot=\{i\}$ ), then the resulting Petri net is strongly connected.

Definition 2 makes some restrictions on workflow-net. Workflow-net must has one beginning point and one endpoint at least. When token enters beginning place, it indicates that instance of process starts. When token enters end place, it indicates that instance of process ends. Workflow-net is a connected graph.

Note that the requirement mentioned in definition 2 is basic requirement. It means that, a workflow-net satisfied definition 2 perhaps leads to deadlock or live-lock.

\section{Modeling for Process of Administrative Law Enforcement}

Taking current regulations in China as example, the administrative law enforcement includes labor arbitration law enforcement, administrative reconsideration law enforcement, the administrative licensing law enforcement and administrative punishment law enforcement, etc. Next discussion is based on administrative reconsideration law enforcement.

Administrative Reconsideration Law of PRC[3] has described the process of administrative reconsideration law enforcement. Having ignored some details that are not important, administrative reconsideration law enforcement consists of five main steps described as follows:

(1) Application of applicant. If applicant who concerns with specific administrative act has specific administrative reconsideration request and reason, he can propose administrative reconsideration application in legal application deadline, in oral or written manner. Application should be submitted to administrative reconsideration institution.

(2) Review and acceptance. Having received application of applicant, administrative reconsideration institution should finish review according the law and make decision to accept or not. Meanwhile, notice of acceptance should be sent to applicant, notice of replication should be sent to respondent.

(3) Declaration of party. Having received notice, both of the parties should according to oneself notice submit material outline, evidence material, letters of authorization, etc. 
(4) Trial of administrative reconsideration institution. Administrative reconsideration institution checks all the material, then make a reconsideration decision.

(5) Settlement of administrative reconsideration. When administrative dispute has been settled or canceled, administrative reconsideration institution will make final decision. In China there are three kinds of settlement, making administrative reconsideration decision, success mediation and terminating administrative reconsideration.

These five steps are only rough description. Detail flows described according to workflow-net specification are as Fig. 1.

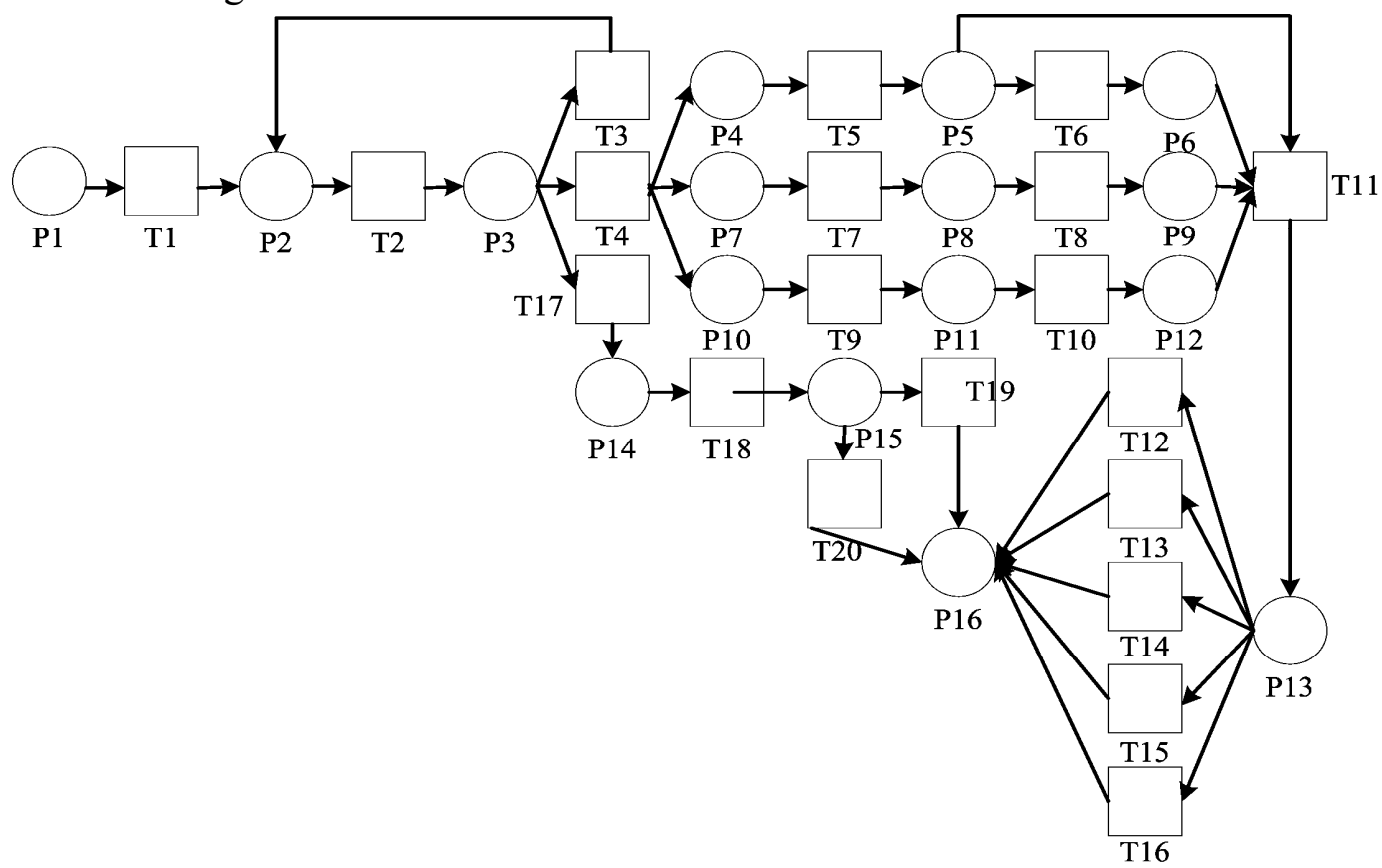

Fig. 1. Workflow-net of administrative reconsideration law enforcement

Transitions in Fig. 1 have different meanings, described as follows:

T1: submitting administrative reconsideration application

T2: review and acceptance

T3: correction of all material

T4: deciding to accept

T5: looking for third party

T6: the third party supplying material

T7: writing and sending reply notice

T8: the respondent submitting material

T9: writing and sending acceptance notice

T10: the applicant submitting material

T11: trial

T12: achieving reconciliation agreement

T13: achieving mediation agreement

T14: writing and sending decision paper

T15: pause of trial

T16: terminating trial

T17: rejecting

T18: asking whether the applicant applying for relief

T19: the applicant applying for relief

T20: the applicant do not apply for relief 


\section{Simplifying the Model}

Administrative reconsideration law enforcement is not most complex; labor arbitration law enforcement is more complex in China administrative laws. Workflow-nets for them all are too complex to verify. Keeping original attributes of nets as a premise, these nets should be simplified. Simplification technology proposed by Murata[4] is used here. There are six simplification rules: fusion of series places, fusion of series transitions, fusion of parallel places, fusion of parallel transitions, elimination of self-loop places, elimination of self-loop transitions. The technology is illustrated as Fig. 2.

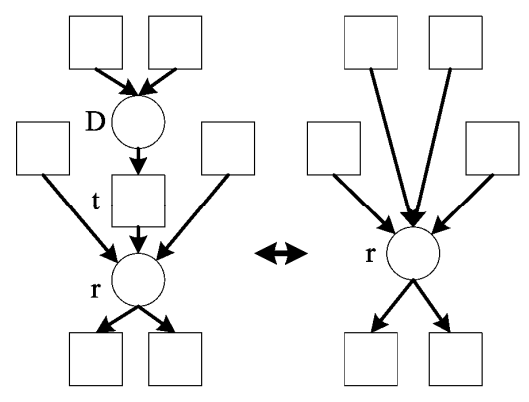

Fusion of Series Places

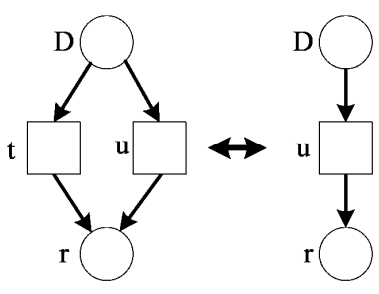

Fusion of Parallel Transitions

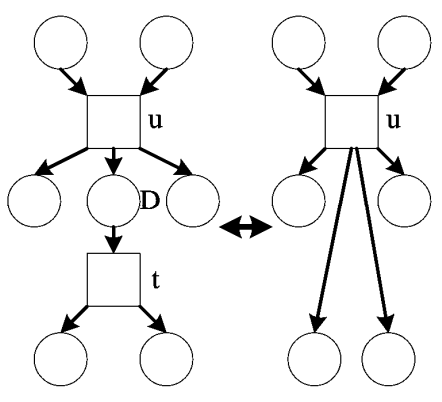

Fusion of Series Transitions

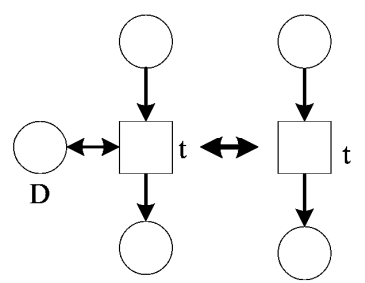

Elimination of Self-loop Places Elimination of Self-loop Transitions

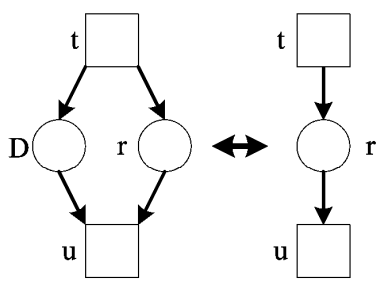

Fusion of Parallel Places

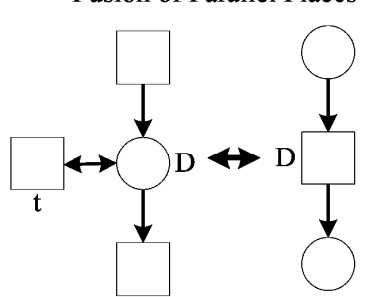

Fig. 2. Simplification rules proposed by Murata

Using Murata's technology, Fig. 1 is reduced to Fig. 3. All original attributes of workflow-net for administrative reconsideration law enforcement are reserved; a simpler net is help to verify itself.

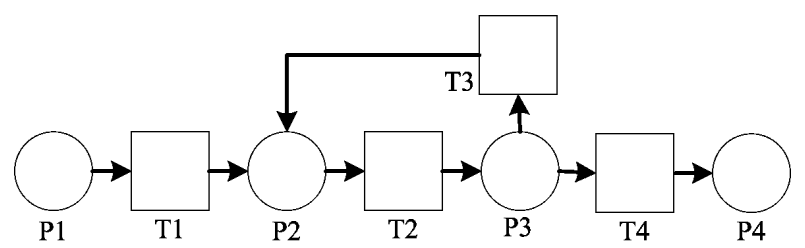

Fig. 3. Simplified workflow-net for administrative reconsideration law enforcement

\section{Verification of Workflow-net}

Soundness of workflow-net is crucial. A workflow-net with error or defect can lead to unpredictable result, such as deadlock or live-lock; process of business will not be executed correctly. So workflow-net must pass verification before execution.

A workflow-net is sound if and only if it meets three requirements:

(1) There is and only one token occurred in sink place at last, which come from source place.

(2) When token occurred in sink place, all the other places are empty.

(3) Every transition can reach all ready status of itself from beginning status.

The first requirement denotes that every instance can be finished successfully. The second requirement denotes that instance having been finished cannot be referenced. These two requirements mean that there is only one final status that only a token exists in sink place. The third requirement denotes that there is no dead task in work flow, namely every task has possibility of execution in principle. 
According to description of soundness, we can follow next steps to verify whether a work flow model is sound or not. These steps are listed as follows:

(1) Confirm that a workflow-net has and only a beginning place and a sink place.

(2) Confirm that there are no free tasks and conditions, namely every task or condition lives in the path from beginning place to end place.

(3) Confirm that every work flow instance will be finished at last.

(4) Confirm that the others places are empty when a token occurred in end place.

(5) Confirm that every task has the possibility of execution, namely there is no dead task.

Simplified workflow-net for administrative reconsideration law enforcement, described by Fig. 3, can be verified through these five steps. In Fig. 3, there is only a beginning place P1 and a sink place P4. Every node in Fig. 3 is connected, namely there is no free task or condition. But there is dead loop in Fig. 3, it means that some work flow instances perhaps cannot be finished and some tasks in Fig. 3 have no chance to be executed along a certain path. So workflow-net illustrated by Fig. 3 is not sound.

Why the net is not sound? Administrative reconsideration law of the PRC has follow provision: if the applicant has not provided sufficient material, he will be asked for correction of all material. But repeating times is not limited. In judicial practice, the course may be endless.

\section{Summary}

Using model technology based on workflow-net, processes of administrative law enforcement are tidied up, and work flow nets are drawn. These nets can be verified and simulated. If there are problems excluding comprehension error, such as soundness shortage, we can manage to improve it by software technology, but we cannot modify it. Because we cannot modify laws, absence of legal prohibition means freedom. Simplified workflow-net for administrative reconsideration law enforcement, described by Fig. 3, has been proved soundless. In our program realization, we keep the loop, but give teacher administrator right. If teacher finds students always circle in the loop, he will think it as malicious action then terminates the experiment and give low score.

Models of administrative law enforcement with workflow-net technology have accurate semantics and intuitive graphs, can be analyzed and optimized conveniently. It helps to find problem and improve it in program.

\section{REFERENCES}

[1]Wil van der Aalst, Kees van Hee. WorkFlow Management Models, Methods, and Systems. Beijing: Tsinghua University Press, 2004。

[2] W. d. v. van der Aalst. The application of petri nets to workflow management. The Journal of Circuits, Systems and Computers, 8(1):21-66, 1998.

[3] State council of PRC. Administrative Reconsideration Law of PRC. Law Press, Beijing, 2009

[4]Murata T. Petri Nets: Properties, Analysis and Applications. Proceedings of the IEEE, 1989,77(4):541-580. 